\title{
Carnets
}

Revue électronique d'études françaises de l'APEF

Deuxième série - 7| 2016

Plurilinguisme et migrations dans la littérature de langue française

\section{Parcours identitaire au travers des langues dans Le bleu des abeilles de Laura Alcoba}

\section{Orianne Guy}

\section{CpenEdition}

Journals

Édition électronique

URL : http://journals.openedition.org/carnets/1046

DOI : $10.4000 /$ carnets. 1046

ISSN : 1646-7698

Éditeur

APEF

Référence électronique

Orianne Guy, « Parcours identitaire au travers des langues dans Le bleu des abeilles de Laura Alcoba », Carnets [En ligne], Deuxième série - 7 | 2016, mis en ligne le 31 mai 2016, consulté le 19 avril 2019.

URL : http://journals.openedition.org/carnets/1046 ; DOI : 10.4000/carnets.1046

Ce document a été généré automatiquement le 19 avril 2019

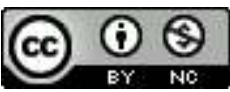

Carnets est mis à disposition selon les termes de la licence Creative Commons - Atribution - Pas d'utilisation commerciale 4.0 International. 


\title{
Parcours identitaire au travers des langues dans Le bleu des abeilles de Laura Alcoba
}

\author{
Orianne Guy
}

\section{Introduction}

1 En 2013, les éditions Gallimard publient Le bleu des abeilles. On pourrait qualifier ce roman d'autofiction car le récit s'inspire largement de l'histoire de son auteure Laura Alcoba. Il s'agit ainsi de l'expérience d'une fillette argentine âgée de dix ans qui rejoint sa mère exilée en France durant la dictature (1976-1983). Son arrivée au Blanc-Mesnil, ville située à côté de Paris, a lieu après deux années d'attente et de préparation linguistique, en janvier 1979. Tout au long des dix-huit chapitres, la voix narrative autodiégétique, celle de l'enfant, nous raconte son rapport à l'exil au travers des langues espagnole et française.

2 Cette relation aux langues semble particulièrement intéressante car, au-delà du récit, l'auteure écrit ses textes en français. D'abord langue-relais, le français est très vite devenu sa langue maternelle de seconde origine ${ }^{1}$. Pour Laura Alcoba, une certaine idéologie linguistique ${ }^{2}$ 'est opérée quant à l'espagnol qu'elle décrit comme étant la langue dans laquelle elle a dû se taire en Argentine. Elle dit à son sujet :

C'est une langue qui m'émeut beaucoup, que j'aime énormément lire. Mais quelque chose reste lié pour moi aux années de dictature. L'espagnol, c'est la langue dans laquelle j'ai eu peur. C'est la langue dans laquelle j'ai appris à me taire. Je me sens plus détendue, plus libre en français, sans doute (Alcoba in Ducrozet, 2014).

Dans son premier roman Manèges, petite histoire argentine (2007), le lecteur découvre cette réalité subie par une fillette qui pourrait être la même que dans Le bleu des abeilles, mais cette fois-ci en Argentine. Nous découvrons ainsi une enfant silencieuse qui endosse des responsabilités bien trop importantes pour son âge car elle risquerait de mettre en danger ses parents et leur réseau opposés à la dictature. D'un point de vue 
psychanalytique, cet acte de refus de la langue maternelle est considéré, selon l'anthropologue Francis Zimmerman, comme un "mécanisme de refoulement». Nous nuançons toutefois cette affirmation car Laura Alcoba écrit des articles en espagnol mais préfère la langue française pour ce qui a trait à sa production littéraire.

La réception de ses œuvres pose la question d'une appartenance nationale. En effet, l'auteure est considérée comme écrivaine argentine dans son pays d'origine alors que ses textes sont écrits en français ${ }^{3}$. Par ailleurs, Laura Alcoba est invitée tant à l'occasion de salons de livres français qu'argentins ; lors d'un entretien, elle exprime ressentir de la joie quand on l'invite en tant qu'écrivaine française $e^{4}$. Rappelons ici qu'elle est naturalisée française depuis ses quinze ans. Pour l'auteure, c'est la langue française qui lui a permis d'écrire des romans ${ }^{5}$. De plus, elle a préféré ne pas traduire elle-même ses œuvres mais en a laissé le soin à l'écrivain Leopoldo Brizuela. Elle raconte à ce sujet : «J'étais aux aguets. Il fallait que ce soit exactement pareil. Que ça sonne identique. Alors que j'aurais bien été incapable de les écrire en espagnol " (Alcoba in Ducrozet, 2014). Lors d'un entretien, Laura Alcoba rappelle la demande décalée qui lui a été formulée à un salon du livre à Francfort ${ }^{6}$ : on lui demandait de lire un passage de son roman en espagnol alors que sa langue d'écriture est le français. Cette littérature fait partie d'un « entre-deux cultures »: une langue, le français, une histoire, l'exil argentin.

Concernant la "nationalité » d'une œuvre, Pascale Casanova perçoit une culture qui s'affranchit des frontières : celle des lettrés, des «législateurs (...) de la République des Lettres ». Cette société est « une et indivisible » car elle est " nationale et internationale » (Casanova, 1999 : 37-38). Analogue à cette absence de frontière dans le domaine littéraire, la culture d'un pays se soustrait, elle aussi, à des barrières physiques. Pour Pilar Blanco García " la cultura es viajera, no se detiene en el país cuando sus habitantes se van. » (Blanco García, 2002 : 174). Conséquemment, aucune définition « limitée » ne suffirait à décrire les notions de littérature et de culture.

Si nous revenons maintenant au récit en lui-même, nous pouvons observer deux grands axes qui s'articulent tout au long de l'histoire, mettant en exergue le rapport de la protagoniste aux langues. D'une part, le lecteur est face à une véritable réflexion sur la langue en elle-même et sur le rapport qu'un individu peut tisser avec elle. Pour Francis Zimmerman « la situation de l'exil est une situation privilégiée pour observer l'aliénation, la privation de sa langue maternelle » qu'il définit comme étant la « langue dont on a été privé, dont on doit faire le deuil » (Zimmerman, 2015). D'autre part, la langue est vécue comme un lieu où l'exilé trouverait un espace où reconstruire sa vie et s'« enraciner ». Laura Alcoba parle pour ce qui la concerne d' « un voyage dans la langue française »"

\section{Réflexions sur le rapport à la langue}

7 Selon l'auteure, apprendre une langue se fait à la fois dans la tête et dans le corps ${ }^{8}$. La protagoniste de son roman cherche à s'approprier le français de cette façon et partage ses réflexions au sujet de cet apprentissage qu'elle espère réussir. Dès les premières pages, nous lisons la langue comme un phénomène phonologique étudié par une fillette : « Avec Noémie, j'ai découvert des sons nouveaux, un $r$ très humide que l'on va chercher tout au fond de la gorge, et des voyelles qu'on laisse résonner sous le nez, comme si on voulait à la fois les prononcer et les garder un peu pour soi » (Alcoba, 2013: 12). Concernant certaines prononciations, la protagoniste explique qu'il faut leurrer son corps, ce qui revient à une sorte de dédoublement : «Il faut faire croire à ses lèvres qu'on va dire une 
chose et en dire une autre. Au début, c'est comme si on leur tendait un piège " (Alcoba, 2013 : 38). Pour s'approprier cette nouvelle langue, la fillette donne le sentiment de se détacher de son corps trop empreint de la langue espagnole. Son corps doit se défaire de ses repères linguistiques appartenant au passé pour laisser place aux repères du présent. Enfin, à la toute fin du récit, la protagoniste révèle qu'elle s'est «souvent demandé comment ça se passait dans la tête des gens qui parlent français depuis toujours " (Alcoba, $2013: 115$ ) ; il est question ici du mécanisme mental du langage.

Outre ces appréciations et réflexions qui jalonnent le récit, la narratrice nous fait part des échanges épistolaires qu'elle entretient avec son père emprisonné en Argentine. Ce lien avec le présent qui lui a été volé permet une éducation à distance ${ }^{9}$. En effet, cette relation se base sur des dialogues et débats -en décalé- à propos de livres communs que père et fille lisent de chaque côté de l'océan Atlantique ${ }^{10}$. Ceci permet à la fillette de faire connaissance avec la langue française par écrit et de préserver l'espagnol écrit, car les courriers reçus à la prison étaient surveillés et aucun mot étranger ne devait apparaitre. Le livre dont il est principalement question dans le roman de Laura Alcoba s'intitule La vie des abeilles de Maurice Maeterlinck, titre qui rappelle celui du roman en possession du lecteur (Alcoba, 2013 : 23). Parallèlement à ce lien, la fillette s'impose un planning à respecter pour envoyer des lettres à ses amis et membres de la famille restés en Argentine. Nous voyons au travers de ces mises en abîme de l'écriture que la langue écrite fait partie du quotidien de l'enfant et revêt une importance dans la construction du personnage. Son rapport avec la langue française débute avant son départ lorsqu'elle prend des cours de langue pour se préparer à rejoindre sa mère. Elle découvre alors avec enthousiasme l'accent grave et le circonflexe ainsi que la lettre ç qu'elle a « tout de suite aimé[e] » (Alcoba, 2013 : 12) ; là-bas, elle s'entraînait à écrire le mot « français » ou encore à faire des chaîne de ç. Cette première approche est ressentie comme esthétique.

9 Par-delà le rapprochement progressif de la fillette avec la langue française, s'effectue une véritable quête de relation d'intimité avec cette langue. Dans Les exilés du langage, AnneRosine Delbart affirme que «[1]a disparition de l'idiome originel ou l'étroitesse de son champ d'application (le cercle familial) peuvent développer une passion exacerbée pour la langue d'accueil devenue l'outil de l'expression écrite » (Delbart, 2005: 68). Ceci se confirme dans Le bleu des abeilles au travers de deux faits : la honte de son accent espagnol et l'envie de réussir son «immersion». Pour l'auteure, parvenir à ne plus avoir d'accent c'était " un habit jeté sur le passé $»^{11}$ et ce désir se retrouve à plusieurs endroits dans le texte lorsque la narratrice répète qu'elle a honte de son accent à cause duquel on la reconnaissait, ce qui la renvoie à sa condition d'enfant exilé, à son passé vécu dans la peur, à son présent perdu « là-bas». Lors de ses lectures, la fillette va préférer certains mots en français à leur traduction en espagnol. En voici un exemple: «'Au seuil de la ruche', ce qui est plus étrange à mes oreilles, mais tellement plus joli, au fond » (Alcoba, 2013 : 24). En contrepartie de la honte ressentie à cause de son accent, la protagoniste connaît le sentiment de fierté : fière d'aller dans une école "française ", fière d'avoir une copine française avec un « français de source » (Alcoba, 2013: 58).

10 Parfois, elle ne cherche pas à comprendre le sens de ce qu'elle entend mais elle s'imprègne de la musicalité de la langue. Nous pouvons ainsi lire :

Je peux rester longtemps, comme ça, à me laisser bercer par la musique de la langue française -je lâche prise du côté des paroles pour ne m'intéresser qu'à la mélodie, aux mouvements des lèvres de tous ces gens qui arrivent à cacher des voyelles sous leur nez sans effort aucun, sans y penser, et hop, -an, -un, -on, ça paraît si simple, en, -uint, -oint : j'écoute, j'admire, j'apprécie (Alcoba, 2013 : 55). 
11 Cette admiration se transforme en fascination pour les e muets. Concernant ces lettres, nous découvrons des exclamations et un champ lexical de l'émerveillement et de l'affection tels les termes "surprise », " abasourdie ", " exaltée », "j'ai aimé », "je les admire », "m'émeuvent» (Alcoba, 2013: 73). Ce qui la touche dans ces lettres c'est qu'elles sont «à la fois indispensables et silencieuses » (Alcoba, 2013 : 73). Nous assistons ici à une assimilation et aussi à une personnification de ces lettres muettes. La fillette ressent une sorte de connivence silencieuse avec ces $e$ muets : «Parfois, j'imagine que les voyelles muettes me voient aussi » (Alcoba, 2013 : 73) ; «J'aime nous imaginer dans cette communication silencieuse » (Alcoba, 2013: 73). Cette relation instaurée préfigure une évolution chez la fillette qui commence alors à s'approprier la langue française au travers de ce qui la différencie de la langue espagnole.

Ainsi, elle commence à s'affirmer d'un point de vue identitaire grâce à ces e muets (« Qu'à notre façon nous sommes ensemble ", Alcoba, 2013: 74) et lors de l'épisode avec la bibliothécaire, la protagoniste prend position face à l'Autre car elle s'investit dans une langue où elle peut avoir une place dans le dialogue donc dans le rapport à l'Autre. À ce moment du récit, la fillette est bien décidée à lire de nombreux textes français. La lecture ne devient plus un acte proposé par le père mais une volonté propre à l'enfant qui souhaite améliorer son français. L'évolution du personnage s'accompagne d'une évolution physique car la fillette devient una señorita (Alcoba, 2013: 89). Ce changement est significatif car le terme est écrit en espagnol dans le texte. Le personnage ne s'est pas rendu compte de son évolution, c'est-à-dire du temps qui passe, de son immersion. Nous lisons: «(...) peut-être parce que, sans m'en rendre compte, j'étais déjà en train de devenir quelqu'un d'autre. Una señorita, c'est ce qu'on dit » (Alcoba, 2013 : 90-91).

Enfin, concernant le rapport à la langue dans l'évolution du personnage principal, nous découvrons que son « bain linguistique » est réussi car, lorsqu'elle séjourne dans les Alpes avec des Français, elle arrive à communiquer sans difficulté avec l'autre fillette prénommée Valérie (Alcoba, 2013 : 95), et s'abstient de parler en espagnol avec Eduardo, un autre enfant réfugié venu du Chili. Dans la montagne, elle découvrira un mot parfait : le mot « reblochon » " avec son $e$ presque éteint dans la première syllabe et sa finale qui vient se placer pile poil sous le nez (...)»(Alcoba, 2013 : 101).

\section{La langue vécue comme un lieu}

14 Avant le départ pour la France, la langue est vécue comme un marqueur de temps puisque la fillette apprend à donner son âge en français. Le lecteur découvre ainsi la durée de l'apprentissage au travers de la langue. Pareillement, l'imaginaire de l'espace au travers de la langue est perceptible dès le début du roman, avant même le départ de la protagoniste car son apprentissage est le point de départ de son exil. Nous relevons les extraits suivants : "J'étais encore en Argentine quand je me suis mise en route. Je ne sais plus si c'est mon grand-père qui m'a annoncé que j'allais bientôt prendre des cours de français » (Alcoba, $2013:$ 11) ou encore «(...), même si mon départ était toujours différé, je me suis mise en route. Quelque part sous mon nez » (Alcoba, 2013 : 15). Par ailleurs, la langue prenait une place dans l'imaginaire de la fillette car elle créait des histoires avec des personnages français inventés. Cette relation à la langue d'un pays inconnu induit une dichotomie spatiale entre le «là-bas » et l'«ici ». Nous lisons en effet: «À chaque cours, dans la salle à manger de mes grands-parents, à La Plata, deux fois par semaine et 
durant près de deux ans, nous nous sommes transportés là-bas -c'est-à-dire ici. » (Alcoba, $2013: 16)$.

Cette conception spatiale de la langue se retrouve au cours du récit au travers de métaphores et de comparaisons. Ainsi, quand elle parle du français de «source » de son amie Astrid, l'instance narrative écrit « Allez savoir jusqu'où plongent les racines de cette langue-là » (Alcoba, 2013 : 58). Les mots, quant à eux, remplissent " un grand espace autour d'eux» et "les syllabes s'incrustaient dans l'air froid comme des cailloux scintillants " (Alcoba, 2013: 98). Dans son article, Sylvie Courtine-Denamy reprend cette notion de spatialité de la langue en parlant de " patrie » : «Dans les trois cas envisagés, la difficulté consiste à se détacher ou à se rattacher à la langue comme s'il s'agissait d'une 'patrie'"; plus loin dans son étude, concernant Hannah Arendt, elle écrit «Les retrouvailles avec la langue maternelle valent donc à ses yeux comme des retrouvailles avec le pays d'origine, elles revêtent une dimension politique et affective " (CourtineDenamy, 2012).

16 Nous revenons maintenant sur la notion de dichotomie entre deux espaces qui sont délimités par les langues. En effet, tandis que sa relation épistolaire avec son père engendre un va-et-vient permanent entre les deux langues, entre son présent perdu en Argentine et son présent imposé en France, sa mère met tout en œuvre pour que la fillette réussisse à s'intégrer à la société au travers de la langue française. Toutefois, dans l'intimité, mère et fille conversent en espagnol. Notons ici que les propos de la maman de la protagoniste ainsi que ceux des autres exilés argentins sont rapportés en italiques, soit en français, soit en espagnol. De ce fait, il n'existe pas réellement de frontière linguistique dans la lecture et le lecteur ne sait pas réellement quelle est la langue parlée. Le discours de la narratrice, qui est le personnage en construction, nous révèle au travers de cette absence de frontière linguistique la porosité des langues. À ce moment du récit, la protagoniste se situe entre deux langues, entre deux réalités. Parallèlement, nous lisons une absence de réaction face à une requête de son père : qu'elle lui choisisse une photo de sa vie en France, sur laquelle elle pose avec sa mère. Or, la fillette fait un blocage avec cette dernière photo à laquelle son père a droit dans sa cellule. Comment interpréter cette réaction? La protagoniste se torture l'esprit, car l'angoisse de ce qui se trame « làbas " arrive jusqu'à elle : la dictature va au-delà des frontières et investit son quotidien avec la censure d'une quelconque trace de mot étranger dans ses courriers. Par ailleurs, elle semble être dans l'impossibilité de partager son présent « ici » car elle ne sait rien de « là-bas ». Nous découvrons à cet effet une multitude d'interrogations (Alcoba, 2013 : 51). Par la suite, la fillette anonyme fait comme si cette requête n'existait pas : serait-ce dans le but de ne pas penser à l'absence de liberté « là-bas »? La dictature s'impose à elle déjà dans le fait de devoir traduire les extraits de textes français en espagnol, mais avec cette photo, la fillette se renferme, reste dans le silence, et a désormais peur de lire le courrier de son père qui est dans l'attente de cette image. Hasard ou non, une fois le « déclic » de la langue effectué, c'est-à-dire quand la fillette commence à s'approprier la langue française, elle est prête à partager une photo d'elle "enracinée » dans cette image qui n'est plus imaginaire mais réelle. Nous pouvons déduire de cet acte qu'il y a eu « enracinement » dans la langue.

L'article va désormais s'orienter vers le mouvement progressif de "déracinement/ enracinement» qui s'est produit dans les langues espagnole et française. Nous avons précédemment observé une situation d'«entre-deux» langues dans laquelle le personnage évolue. Toutefois, si nous nous arrêtons sur certains passages du roman, nous 
pouvons décrypter un changement de position de la fillette par rapport à ces deux langues. Ainsi, un premier détachement est identifiable lorsqu'elle donne la traduction du titre du roman en espagnol. Nous lisons: "Dans ses lettres, mon père recopie, en espagnol, des passages entiers de La vie de abeilles - La vida de las abejas, comme il dit " (Alcoba, 2013: 24). Nous l'avons déjà vu, la fillette est contrainte de se placer entre les deux langues: dans ses lectures mais aussi en écrivant. Elle est contrainte de se placer comme traductrice à seulement 10 ans; au début, elle a du mal à comprendre puis la lecture devient plus fluide car elle travaille son français en effectuant des recherches actives sur du vocabulaire inconnu et spécifique. Nous découvrons la honte de son accent car elle «n' $[\mathrm{a}]$ pas envie qu'on [la] repère » (Alcoba, 2013 : 36). La narratrice raconte : «Cet accent, j'aimerais l'effacer, le faire disparaître, l'arracher de moi » (Alcoba, 2013 : 37) car il lui renvoie le regard des autres, c'est-à-dire sa différence, mais cela lui renvoie aussi à ses origines c'est-à-dire l'Argentine, vécue comme pays de la peur et comme un présent manqué. Pour s'améliorer, elle s'entraîne devant un miroir pour prononcer au mieux ces nouvelles sonorités (Alcoba, 2013 : 37). La fillette veut implanter cette nouvelle langue dans sa bouche et sous son nez afin de retirer toute trace d'existence de l'espagnol quand elle parle français. La narratrice utilise à ce moment un vocabulaire lié à la peur, à la honte, à la persécution, autrement dit un champ lexical renvoyant à son vécu outreAtlantique. À partir de cette volonté de se détacher de son accent, c'est-à-dire d'une partie de son identité, la protagoniste cherche à investir la langue française : «C'est que le bain [linguistique] ne me suffit plus, je veux aller bien plus loin: me trouver à l'intérieur de cette langue, pour de bon, je veux être dedans » (Alcoba, 2013 : 55). S'ensuit une personnification des voyelles et sa prise de conscience d'une capacité non encore révélée. Au moment d'écrire à son père au sujet d'un nouveau livre qu'elle a trouvé en France, elle sent que son titre, Les fleurs bleues de Raymond Queneau, ne rendrait pas la même chose en espagnol. Ce problème de la traduction renvoie ici directement à l'expérience de Laura Alcoba par rapport à ses œuvres. Son rapport avec la lettre $e$ muette la mène à se sentir en "connivence avec l'orthographe française " (Alcoba, 2013: 73). Enfin, au bout de plusieurs mois, elle se demande «quelle distance [la] séparait encore d'un français, qui serait pleinement [sien] »; « Est-ce que j'y arriverai un jour, alors que ça fait si longtemps que je me suis mise en route? " (Alcoba, 2013 : 96). Dans cet extrait, le désir de s'approprier la langue française est explicite et l'expression «un long chemin à parcourir " prend tout son sens dans cette expérience d'une acquisition d'une langue étrangère. Puis, la fillette se demande «Par où ça passe? ? (Alcoba, 2013 : 116) car elle veut vraiment s'approprier les mots de la langue française. Elle recherche alors la fluidité qu'ont les gens qui parlent cette langue depuis toujours. Elle cherche le «bon tuyau » car, pour elle, cela se passe en deux temps : elle pense en espagnol puis traduit en français. Puis, arrive le jour où «J'ai pensé et parlé en français en même temps » (Alcoba, 2013 : 117), à la surprise de sa mère. Le personnage principal est entre la veille et le sommeil, c'est-àdire en « lâcher prise » de son corps et de son esprit. À cet instant, elle s'adresse à sa mère en français et reste "émerveillée et effarée à la fois » (Alcoba, 2013 : 118). La narratrice déclare: «J'avais trouvé l'ouverture » (Alcoba, 2013: 118), c'est-à-dire le « déclic » de la langue, le « tuyau » où se faufiler. Le fait de s'être approprié cette langue qui a pris corps en elle lui permet d'accepter sa réalité " ici ", sans son père, à qui elle envoie la cinquième photo par la suite. 


\section{Conclusion}

18 Cette étude nous amène à conclure que l'écriture et l'expérience de Laura Alcoba nous révèlent l'harmonie avec laquelle une langue, maternelle ou " adoptée ", peut investir le corps d'un individu, sa personnalité, mais aussi le guider dans sa construction individuelle et au sein de la collectivité, face aux autres. Le rapport aux langues est vécu de façon plus ou moins complexe selon les situations d'exil, selon les personnalités. Pour l'auteure en question, le français semble être ressenti comme une " patrie de la liberté » où elle enracine ses écrits littéraires.

\section{BIBLIOGRAPHIE}

BAREIRO SAGUIER, Rubén (1989). « Escritura y exilio », in Gilberto de León (Coord.), Novela y exilio : Entorno a Mario Benedetti, José Donoso, Daniel Moyano. Montevideo : Editorales Signos, pp. 17-23. BLANCO GARCIA, Pilar (2002). «La transculturidad de los escritores exiliados », in Béatrice Cáceres et Yannick Le Boulicaut (Coord.), Les écrivains de l'exil : cosmopolitisme ou ethnicité. Paris :

L'Harmattan, Coll. « Cahiers du CIRHILL », n² 25, pp. 173-190.

CASAnova, Pascale (1999). La République Mondiale des Lettres. Paris : Ed. du Seuil.

COURTINE-DENAMY, Sylvie (2012). « Entre Deux Langues. Langues et affect » Item [on-line] (mis en ligne le 04/06/2012) [disponible le 29/01/15]

<URL : http://www.item.ens.fr/index.php?id=578199>.

DELBART, Anne-Rosine (2005). Les exilés du langage, « Un siècle d'écrivains français venus d'ailleurs (1919-2000)». Limoges : Presses Universitaire de Limoges.

DUCROZET, Pierre (17/03/14). « Une histoire argentine » [on-line]. [disponible le 29/01/15]

<URL : http://www.ifverso.com/fr/content/une-histoire-argentine>.

DIATKINE, Anne (04/08/14), «Le blocage espagnol de Laura Alcoba » Le monde [on-line] [disponible le $29 / 01 / 15]$

$<$ URL : http://www.liberation.fr/livres/2014/08/04/le-blocage-espagnol-de-laura-

alcoba_1075473>.

SISCAR, Cristina (1999). « Yo conocí los dos exilios », in Jorge Boccanera (Coord.), Los escritores del exilio, Tierra que anda. Buenos Aires : Ameghino.

ZIMMERMANN, Francis. « Langues de l'exil et de la mémoire » [on-line]. [disponible le 30/01/15], $<U R L$ : http://www.archivesaudiovisuelles.fr/FR/_video.asp?

$\mathrm{id}=341 \&$ ress $=1204 \&$ video $=86814 \&$ format $=68 \# 3174>$. 


\section{Entretiens / Rencontres}

Libération du 4 août 2014. [disponible le 29/01/15]

<URL : http://www.liberation.fr/livres/2014/08/04/le-blocage-espagnol-de-laura-

alcoba_1075473>.

Quinzième édition du festival littéraire franco-irlandais à Dublin. [consulté le 13/02/15]

<URL : https://www.youtube.com/watch ?v =WtbKKVzieY4>.

Émission de France Inter du 23/10/13. [disponible le 13/02/15]

<URL : http://www.franceinter.fr/player/reecouter?play=751776>.

Rencontre écriture et frontières / Laura Alcoba, Antonio Altarriba. [disponible le 13/02/15]

<URL : https://www.canal-u.tv/video/universite_toulouse_ii_le_mirail/

rencontre_ecriture_et_frontieres_laura_alcoba_antonio_altarriba.14348>.

\section{NOTES}

1. Cf. Expression employée par l'anthropologue Francis Zimmermann lors du séminaire « Langues de l'exil et de la mémoire » [on-line] [disponible le 30/01/15]

$<$ URL:

http://www.archivesaudiovisuelles.fr/FR/_video.asp? id=341\&ress=1204\&video=86814\&format=68\#3174>. L'anthropologue rapproche cette réalité à la figure rhétorique appelée « catachrèse » qui signifie donner à un mot, dont on a perdu le sens de l'image première, un sens propre de seconde origine.

2. Voir à ce sujet l'article de Sylvie Courtine-Denamy "Entre Deux Langues. Langues et affect ", dans lequel elle invite à analyser le rapport aux langues de philosophes et écrivains juifs de langue allemande pendant la Seconde Guerre mondiale [on-line] [disponible le 13/02/15] <URL: http://www.item.ens.fr/index.php?id=578199>.

3. Bien que traduits en espagnol par Leopoldo Brizuela, ses livres sont publiés dans la collection dédiée à la littérature argentine dans son pays d'origine.

4. [on-line] [disponible le 13/02/15] <URL: https://www.youtube.com/watch?v=WtbKKVzieY4>, à l'occasion de la quinzième édition du festival littéraire franco-irlandais à Dublin.

5. «Sans le français, je n'aurais pas écrit. C'est l'espagnol qui m'a appris à me taire. En espagnol, enfant, je suis devenue mademoiselle Rien du tout: il m'était interdit de dire comment je m'appelais. On ne se défait pas d'un tel pacte. C'est la langue française qui m'a permis de sonder le silence et de m'en défaire » in Libération du 4 août 2014, [on-line] [disponible le 29/01/15] <URL:http://www.liberation.fr/livres/2014/08/04/le-blocage-espagnol-de-laura-alcoba_1075473 $>$.

6. Rencontre écriture et frontières / Laura Alcoba, Antonio Altarriba, [on-line] [disponible le 13/02/15]

$<$ URL: http://www.canal-u.tv/video/universite_toulouse_ii_le_mirail/ rencontre_ecriture_et_frontieres_laura_alcoba_antonio_alta>.

7. [on-line] [disponible le 13/02/15] <URL: https://www.youtube.com/watch?v=WtbKKVzieY4>, à l'occasion de la quinzième édition du festival littéraire franco-irlandais à Dublin.

8. Idem

9. Rubén Bareiro Saguier, écrivain paraguayen, déclare à ce sujet : « Todos estuvimos amputados : ellos, de la libertad, nosotros, del contexto », BAREIRO SAGUIER, Rubén (1989). « Escritura y exilio », in Gilberto de León (Coord.), Novela y exilio : Entorno a Mario Benedetti, José Donoso, Daniel Moyano. Montevideo: Editorales Signos, p. 22. 
Pour sa part, Cristina Siscar parle d'« amnésie de l'Autre » : «(...), aunque hayamos mantenido contactos permanentes por todos los medios posibles, nuestro exilio es una ausencia en la memoria de los otros, una amnesia. Algo que sucedió en otra parte ", SISCAR, Cristina (1999). « Yo conocí los dos exilios » in Jorge Boccanera (Coord.), Los escritores del exilio, Tierra que anda. Buenos Aires: Ameghino, p. 59.

10. Cette anecdote renvoie à la relation que Laura Alcoba entretenait avec son père et dont elle parle dans un entretien: "Nous avons été séparés pendant très longtemps, or il m'a transmis quelque chose de très important sur la littérature. Il me guidait dans les livres, que je lisais en français, et lui en espagnol ». «Une histoire argentine », publié le 17/03/14, par Pierre Ducrozet, [on-line] [disponible le 29/01/15]

<URL: http://www.ifverso.com/fr/content/une-histoire-argentine>.

11. «En français, il n'y avait plus de censure, tout pouvait être dit. Mais avoir un accent, c'est le début d'une histoire. Il suffisait qu'on me demande l'heure, pour qu'une vie antérieure apparaisse. Parvenir à ne plus en avoir, c'est un habit jeté sur le passé » in Libération du 4 août 2014. [disponible le 29/01/15]

<URL : $\quad$ http://www.liberation.fr/livres/2014/08/04/le-blocage-espagnol-de-lauraalcoba_1075473>.

\section{RÉSUMÉS}

Paru en 2013, Le bleu des abeilles laisse libre parole à une fillette argentine anonyme qui rejoint sa mère exilée en France. Laura Alcoba, auteure de ce roman vivant actuellement dans l'Hexagone, a vécu une expérience similaire et s'inspire de son passé pour narrer ce qui était alors tu pendant la dictature (1976-1983). Là-bas, outre-Atlantique, il fallait se taire pour se protéger; ici, en France, la parole est possible. Ce rapport à la langue assez complexe se révèle aux yeux du lecteur comme un cheminement progressif de la protagoniste ; vécu d'abord comme une contrainte, il devient une libération. Le temps du récit est celui d'une fillette coincée entre deux temps, deux expériences, représentés par ces deux langues que sont l'espagnol et le français. L'étude proposée tâche de dévoiler les stratégies narratives que l'auteure a employées afin de valoriser la place de la langue dans la construction identitaire de son personnage en exil.

Published in 2013, Le bleu des abeilles gives word to an anonymous Argentinean girl who joins her mother exiled in France. Laura Alcoba, author of this novel, lived a similar experience and is inspired by her past to tell what was then kept silent during the dictatorship (1976-1983). Over there, across the Atlantic, it was necessary to keep silence to protect oneself; here, in France, the word becomes possible. This rather complex relationship to language is perceived by the reader as a progressive growth of the protagonist; experienced at first as a constraint, it becomes a liberation. The time of the narrative is the one of a girl stuck between two times, two experiences, represented by the two languages, Spanish and French. The proposed study tries to reveal the narrative strategies which the author used to value the place of language in the identity construction of her character in exile. 
INDEX

Keywords : exile, language, identity, dictatorship, Argentina

Mots-clés : exil, langue, identité, dictature, Argentine

\section{AUTEUR}

ORIANNE GUY

Université de Rennes 2

orianneguy[at]yahoo.fr 